\title{
Precision Hilar Cholangiocarcinoma Surgery
}

\author{
Thomas A. Aloia, MD \\ Department of Surgical Oncology, University of Texas-MD Anderson Cancer Center, Houston, TX
}

Hilar cholangiocarcinoma is one of the most challenging diseases that a hepatobiliary surgical oncologist will treat. In half of the cases there is no preoperative tissue diagnosis, and although the diagnostic accuracy of both computed tomography and magnetic resonance imaging have improved, surgeons frequently do not have a firm handle on the longitudinal extent of tumor spread before exploration. By definition, the vast majority of these resections require extended hepatectomy, which may expand to include the need for concomitant pancreaticoduodenectomy. Even with these radical resections, positive margins occur in as many as $50 \%$ of patients, and the oncologic outcomes after resection are poor.

In this edition of the Annals of Surgical Oncology, Zhang and colleagues examined a retrospectively collected multi-institution database compiled by ten US hepatobiliary centers. ${ }^{1}$ The focus of the analysis was to determine the risks and benefits related to additional biliary and surrounding structure resection in cases where the intraoperative frozen section biliary margin is read as positive. Importantly, they found that the accuracy of intraoperative frozen section was high. In addition, they determined that, of the 58 patients who underwent re-resection, 29 patients or $50 \%$ achieved a final R0 margin. This subset of the re-resection subset was rewarded with median and 5-year survivals comparable to patients with primary R0 margin, suggesting that this should be a common practice.

At face value, this would make oncologic sense and would be contributory data towards a recommendation that all patients with intraoperative R1 margins have an attempt

(C) Society of Surgical Oncology 2018

First Received: 21 February 2018;

Published Online: 8 March 2018

T. A. Aloia, MD

e-mail: taaloia@mdanderson.org at further resection. However, there are some wrinkles in the data indicating the need for thoughtful restraint. We are not provided information on whether the positive margin was mucosal involvement or the more common peribiliary lymphatic involvement. Historically, it has been taught that, in the latter case, "chasing the margin" deep into the liver is futile. In support of this hepatobiliary oncolore, the current study found that perineural invasion was the dominant oncologic prognostic factor [hazard ratio $(\mathrm{HR})=2.2$ ], along with regional adenopathy $(\mathrm{HR}=1.9)$, in contrast to lesser weight of R1 status (HR = only 1.3).

Likewise, it is not well described in the paper what specific re-resections were performed and the magnitude of these re-resections. In this type of surgery, if a hilar Bismuth-Corlette type I tumor is resected and the proximal margin is positive, the surgeon is frequently obligated to an extended hepatectomy with caudate resection, because the entire hilum must be removed. ${ }^{2}$ With the more common types II, III, and IV tumors, proximal margin positivity frequently converts a patient with a single hepaticojejunostomy anastomosis to two or more small and challenging anastomoses as the re-resection crosses segmental biliary radicals deeper within the right and/or left livers. Distally, when initial resection of the mid-bile duct is oncologically optimized down to the level of the pancreatic head and that margin is positive, additional margin control frequently necessitates conversion to a Whipple resection (only performed $1.2 \%$ of the time in this series and not ever in the R1 to R0 conversion group).

In summary, provided that an adequate oncologic resection was initially performed, the subsequent re-resection of the bile duct almost always results in a substantial increase in the magnitude of the operation and a concomitant elevation in risk. However, in the current study, patients in the re-resection R0 group experienced the lowest rates of biliary fistula, anastomotic leak, and mortality. In fact, there were no 30-day mortalities in this group, whereas the overall mortality rate in this entire 
cohort was high at $5.8 \%$. Certainly, preservation of $\sim 6 \%$ of patients in the long-term follow-up buoyed the oncologic outcome numbers in this subset. It is paradoxical that a re-resection, typically obligating a significant increase in surgical complexity, would result in lower short-term complication rates.

The high overall mortality rates combined with several other aspects of the data, including a low caudate lobe resection rate of only $35 \%$ and a low vascular resection rate of only $8.6 \%$, point to a broader issue with the conduct of hilar cholangiocarcinoma surgery in the US. One of the only logical explanations for this constellation of findings is that a large number of patients in this study had an initial limited biliary resection, leaving the surgeons the opportunity to take more biliary margin without substantially increasing the magnitude of the operation or the difficulty of the anastomoses.

During the past three decades, we have learned multiple lessons from our European and Asian colleagues regarding the quality and safety of the surgical management of hilar cholangiocarcinoma. Professors Nagino, Nimura, Bismuth, Neuhaus, and others have demonstrated incredibly low mortality rates after these massive operations, while almost universally performing preoperative biliary drainage, portal vein embolization, and extended hepatectomy to include the caudate lobe with or without concomitant pancreaticoduodenectomy. $^{3-5}$ Their groups have, in particular, emphasized the oncologic and technical importance of including the caudate lobe and en bloc vascular resections in hilar cholangiocarcinoma surgery. ${ }^{6}$

Integrating the current study with the literature on this topic it is likely that three conclusions can be made. First, all hepatobiliary surgeons should strive to have an optimal initial biliary resection extending proximally to include at least the caudate liver and the main right or left ducts, as indicated, and distally down to the cephalad border of the pancreas, to include the surrounding parenchymal structures, portal nodes, and involved vasculature. Second, after this optimal initial resection is performed, if the biliary margin remains positive and additional resection does not substantially increase the complexity of the operation (rare), Zhang and colleagues' data would suggest that this is an acceptable and even advisable practice. Third, when additional resection obligates the surgeon to additional liver parenchymal loss, jeopardizing postoperative liver function and converting the patient to a complex hepaticojejunostomy, and/or necessitates pancreaticoduodenectomy (common), the data from this study may not apply. In these cases, it is likely that surgeons will encounter substantial increases in morbidity and mortality, with rare oncologic benefit, and should proceed with caution.

\section{DISCLOSURES None.}

\section{REFERENCES}

1. Zhang X-F III, Squires MH, Bagante F, Ethun CG, Salem A, Weber SM, et al. The impact of intraoperative re-resection of a positive bile duct margin on clinical outcomes for hilar cholangiocarcinoma. Ann Surg Oncol. 2018. https://doi.org/10.1245/ s10434-018-6382-0.

2. Bismuth H, Corlette MB. Intrahepatic cholangioenteric anastomosis in carcinoma of the hilus of the liver. Surg Gynecol Obstet. 1975;140(2):170-8.

3. Nimura Y, Kamiya J, Kondo S, Nagino M, Uesaka K, Oda K, et al. Aggressive preoperative management and extended surgery for hilar cholangiocarcinoma: Nagoya experience. J Hepatobiliary Pancreat Surg. 2000;7(2):155-62.

4. Bismuth $\mathrm{H}$, Nakache R, Diamond T. Management strategies in resection for hilar cholangiocarcinoma. Ann Surg. 1992;215(1):31-8.

5. Tsao JI, Nimura Y, Kamiya J, Hayakawa N, Kondo S, Nagino M, et al. Management of hilar cholangiocarcinoma: comparison of an American and a Japanese experience. Ann Surg. 2000;232(2):166-74.

6. Neuhaus P, Thelen A, Jonas S, Puhl G, Denecke T, VeltzkeSchlieker W, et al. Oncological superiority of hilar en bloc resection for the treatment of hilar cholangiocarcinoma. Ann Surg Oncol. 2012;19(5):1602-8. 